\title{
Direct writing of silicon gratings with highly coherent ultraviolet laser
}

\author{
Chung-Yen Chao, Cheng-Yen Chen, and Chee-Wee Liu \\ Graduate Institute of Electro-Optical Engineering and Department of Electrical Engineering, \\ National Taiwan University, Taipei 116, Taiwan, Republic of China \\ Yih Chang \\ Department of Mechanical Engineering, Chung Cheng Institute of Technology, Tahis, Taoyuan 335, \\ Taiwan, Republic of China \\ C. C. Yang ${ }^{\text {a) }}$ \\ Graduate Institute of Electro-Optical Engineering and Department of Electrical Engineering, National \\ Taiwan University, Taipei 116, Taiwan, Republic of China
}

(Received 10 July 1997; accepted for publication 2 September 1997)

\begin{abstract}
Silicon gratings with periods from 180 to $550 \mathrm{~nm}$ were fabricated with a laser ablation technique in which the interference fringe from an intense and coherent ultraviolet laser at $266 \mathrm{~nm}$ directly melted silicon surface. The scanning electron microscopy and atomic force microscopy pictures showed that the corrugations were in quite good quality with the depth as large as $70 \mathrm{~nm}$. The measurement of grating period dependence on temperature showed that rapid thermal annealing could release the thermal strains, which were built during the melting and cooling process in laser ablation, and make the grating period variation more regular. Also, with an air gap between the sample and prism surfaces, the fabricated gratings had weaker thermal strains and more regular temperature dependencies. All the measurement results of temperature dependence were consistent with theoretical predictions. (c) 1997 American Institute of Physics. [S0003-6951(97)03943-0]
\end{abstract}

Diffraction gratings formed by periodical corrugations on semiconductors are important devices for the applications to distributed feedback laser operation, wavelength filtering, sensing, etc. ${ }^{1-5}$ The conventional technique of fabricating periodical corrugations includes the steps of patterning with photoresist and etching. Typical techniques for patterning include e-beam lithography and the interference of a coherent light source. Regarding the etching technique, chemical wet etching was often used; however, for fine etching features the reactive ion etching technique was also used. In this letter, we report the results of direct writing of periodical corrugations on silicon with intense coherent ultraviolet laser and the release of the thermal strains in such a grating.

The periodical corrugations were ablated by the interference fringe of the fourth-harmonic of a Q-switched Nd:YAG laser. This laser system, with its laser diode seeding, provided a very narrow spectral width below $250 \mathrm{MHz}$ at 1064 $\mathrm{nm}$. It is believed that the spectrum of its fourth-harmonic at $266 \mathrm{~nm}$ should have about the same width. According to our measurement, the ultraviolet (UV) source is coherent over its pulse duration of $5 \mathrm{~ns}$. Such a coherent UV source provides us with a good tool for forming interference fringe with a period smaller than $200 \mathrm{~nm}$. Our optical setup is shown in Fig. 1. After the two cylindrical lenses, the laser beam of dimensions $2.2 \mathrm{~cm} \times 0.55 \mathrm{~cm}$ was directed onto a prism (made of fused silica) so that the axis of the refracted beam inside the prism exactly hits a corner of the prism. Therefore, one-half the beam is totally internally reflected by the plane surface right to the corner. ${ }^{6}$ As shown in Fig. 1, the two half-beams interfere on the surface left to the corner where we place the semiconductor sample. The sample directly contacts the prism surface.

${ }^{a)}$ Electronic mail: ccy@cc.ee.ntu.edu.tw
In our experiment, we found that with a reasonable power level from our laser system, we could directly ablate $\mathrm{Si}, \mathrm{GaAs}, \mathrm{InP}, \mathrm{GaN}$ and $\mathrm{LiNbO}_{3}$ to form periodical corrugations with grating periods ranging from 180 to $550 \mathrm{~nm}$. Figure 2 shows the scanning electron microscopy (SEM) pictures of a laser-ablated silicon grating with a $470 \mathrm{~nm}$ period. Periodical corrugations with reasonably good quality can be clearly seen. The grating shown in Fig. 2 was fabricated with a laser energy of $9.6 \mathrm{~mJ}$ per pulse, pulse repetition rate $50 \mathrm{~Hz}$ and exposure time $1 \mathrm{~min}$. Figure 3 shows the atomic force microscopy (AFM) picture of this grating. In both Figs. 2 and 3, (a) shows the grating pictures right after the ablation and (b) shows those after they were dipped for $30 \mathrm{~s}$ with $10 \%$ HF. HF can remove the oxide formed on the surface during laser ablation. The corrugation profile is more regular after the removal of oxide. From the AFM picture, we can see that the corrugation depth is about $70 \mathrm{~nm}$. From the patterns in Figs. 2 and 3, it can be seen that the crests in Figs. 2(a) and 3(a) (before HF dip) become troughs in Figs. 2(b) and 3(b) (after HF dip). This is because the silicon oxide at the crests in Figs. 2(a) and 3(a), which was removed with HF dip, extends in depth beyond the trough level on the same figures.

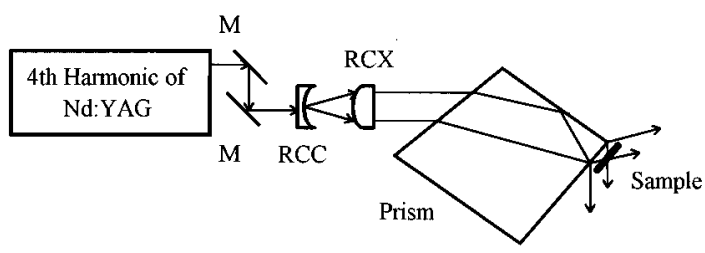

FIG. 1. Experimental setup for forming interference fringe from the fourthharmonic of a Q-switched Nd:YAG laser. Here, M, RCC, and RCX represent mirror, concave cylindrical lens and convex cylindrical lens, respectively. 

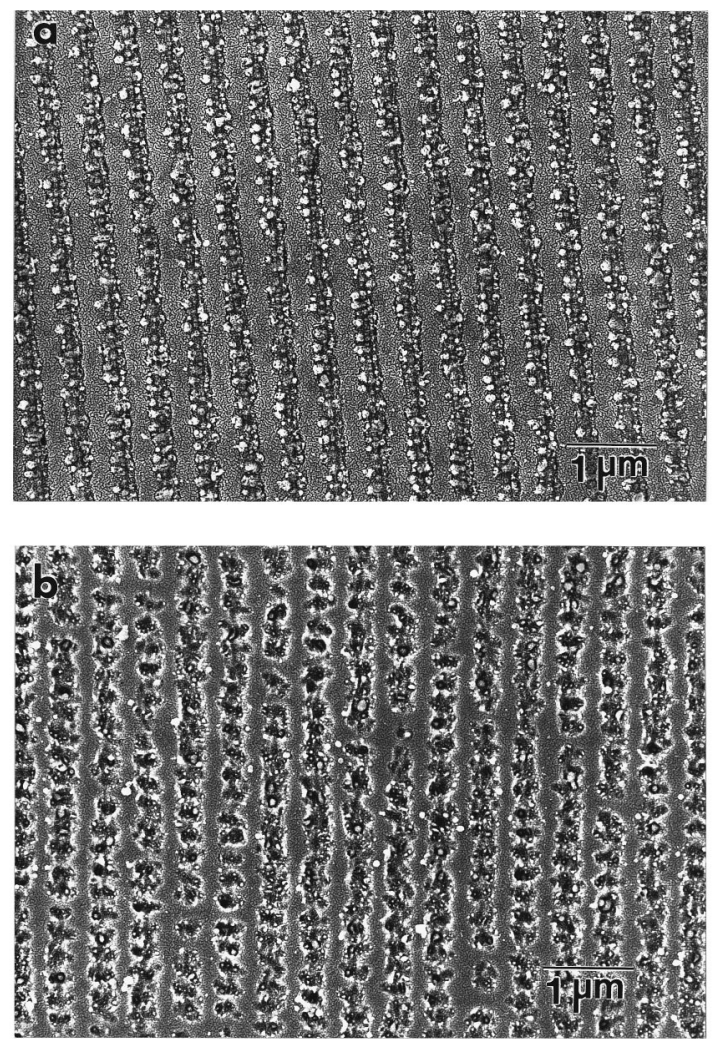

FIG. 2. SEM pictures of a silicon grating with the room-temperature period $470 \mathrm{~nm}$. (a) was taken before HF dip and (b) was taken after HF dip.

The ablation process is hypothesized as follows: During the illumination of a laser pulse, the intense laser of the interference fringe was absorbed and the sample surface was heated at the bright lines to reach a temperature above the melting point of silicon. The molar energy needed for silicon to transfer from the solid phase at $298 \mathrm{~K}$ into the liquid phase at the melting point $(1685 \mathrm{~K})$ is $86.175 \mathrm{~kJ} / \mathrm{mole}^{7}$ In our estimation, with the absorption coefficient of silicon at 266 $\mathrm{nm}$ at $2.09 \times 10^{6} \mathrm{~cm}^{-1}$ and the pulse energy $9.6 \mathrm{~mJ}$, the energy absorbed by 1 mole of silicon is $218.85 \mathrm{~kJ}$ with the illumination of one pulse. Obviously, the laser energy is high enough to melt silicon for fabricating gratings. A part of the melted silicon becomes oxide and forms the crests, as shown in Figs. 2(a) and 3(a). To understand the formation of oxide crests better, we have also ablated silicon with an air gap of $3 \mathrm{~mm}$ between the prism and silicon substrate surfaces. Meanwhile, nitrogen purge was applied during the ablation. We found that in this situation, the oxide crests were lower. This can be attributed to two possible factors. First, less oxygen existed for oxidation with nitrogen purge. Second, without contacting the prism the melted silicon or oxide could be sputtered away from the melting lines.

We have tested the dependence of grating period on temperature with the hope of using such gratings for temperature measurements. In our tests, we monitored the variations of the -1 -order diffraction angle when the silicon grating was heated on a hot-plate. The diffraction angle was calibrated from the beam position on a ruler. To account for the possible tilt of the hot-plate surface during temperature variation, we also monitored the angle of the 0th order diffraction, i.e., the retro-reflection. The calibrated data of the period of a (a)
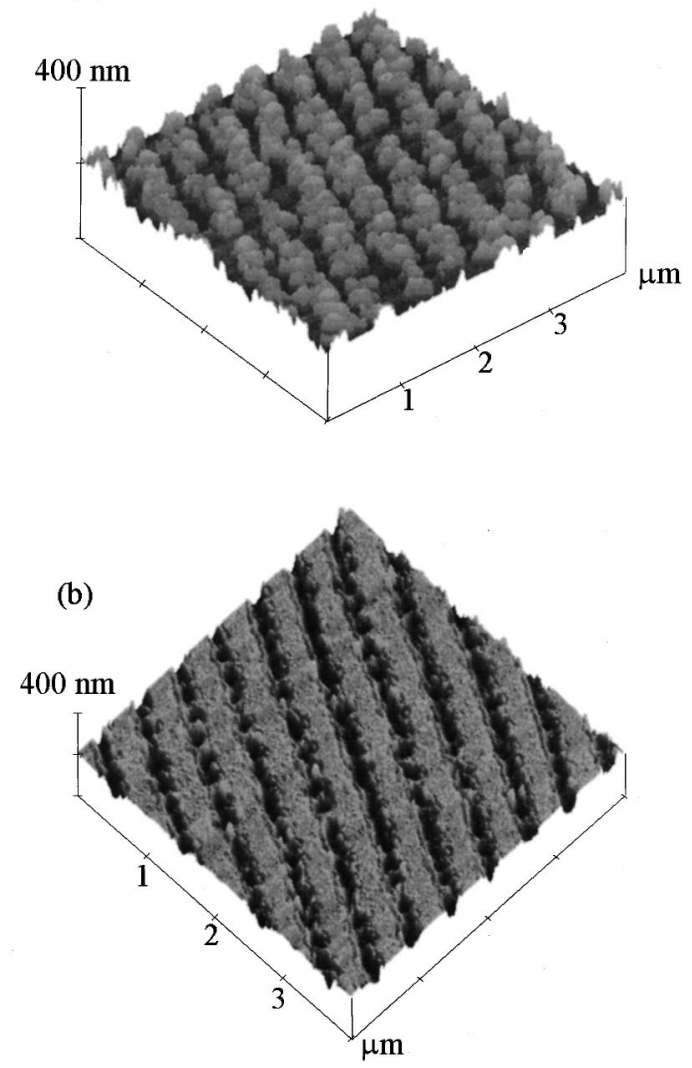

FIG. 3. AFM pictures of the silicon grating shown in Fig. 2. Again, (a) was taken before HF dip and (b) was taken after HF dip.

silicon grating with the room temperature period about 485.3 $\mathrm{nm}$ are shown in Fig. 4. This grating was fabricated in air with silicon substrate directly contacting the prism. The squares represent the data of the grating right after the laser ablation process. We can see that up to $400{ }^{\circ} \mathrm{C}$ the grating period variation is quite irregular. Then, the circles stand for the results after the grating was rapid thermal annealed (RTA) with nitrogen purged at $800{ }^{\circ} \mathrm{C}$ for $30 \mathrm{~s}$. The grating variation becomes more regular with a clear trend over the $800{ }^{\circ} \mathrm{C}$ range. Also, we notice that the room temperature

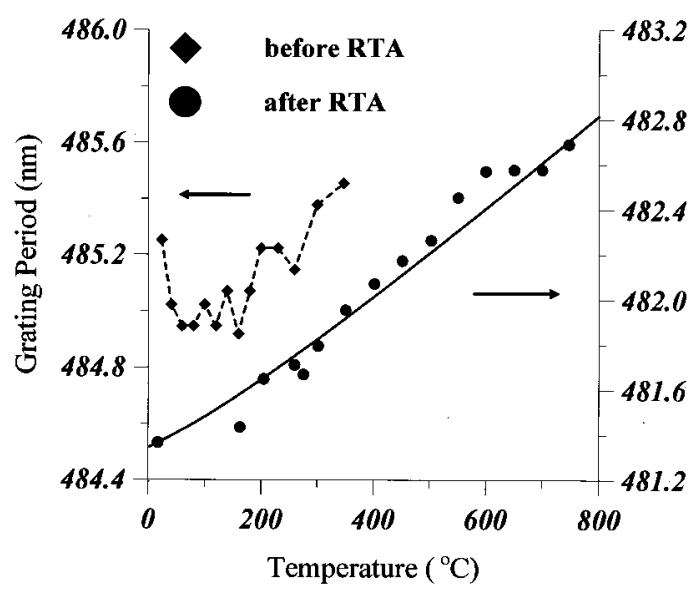

FIG. 4. Grating period as a function of temperature of a silicon grating before and after RTA. The variation of grating period is more regular after RTA. 


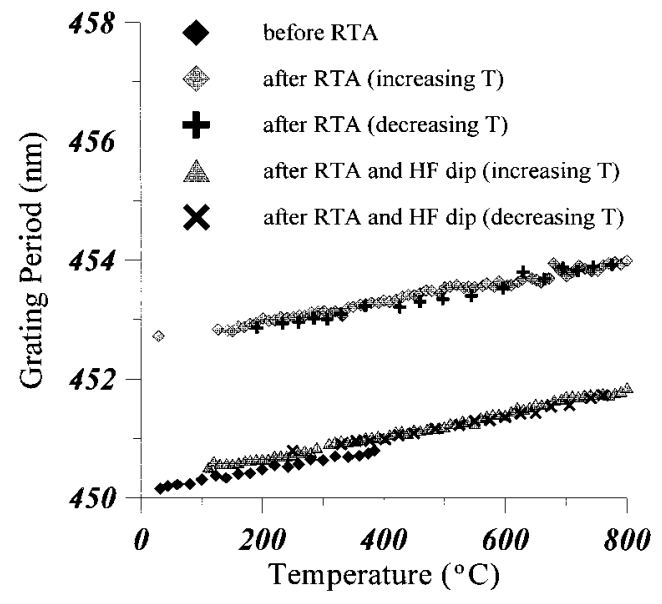

FIG. 5. Grating period as a function of temperature of a silicon grating before and after RTA and HF dip. A gap existed between the sample and prism surfaces during laser ablation which was under nitrogen purge.

grating period has been reduced to about $481.4 \mathrm{~nm}$. The nearly $4 \mathrm{~nm}$ reduction and the more regular behavior after RTA are attributed to the release of the thermal strains built during the laser heating and then cooling process. The reduction of the room temperature period implies that the thermal strain is the tensile type. The solid curve in Fig. 4 represents the theoretical result based on the empirical data. ${ }^{8,9}$ The experimental data are quite consistent with the theoretical prediction.

Figure 5 shows a set of results under the condition of laser ablation with the air gap and nitrogen purge. The dark diamonds represent the measurement results before RTA up to $400{ }^{\circ} \mathrm{C}$. The variation is quite smooth compared with the corresponding data in Fig. 4. The relatively better result in Fig. 5 implies that the aforementioned thermal strain is weaker when less oxide exists. The gray diamonds and crosses in Fig. 5 stand for the results after nitrogen-purged RTA $\left(800{ }^{\circ} \mathrm{C}\right.$ for $\left.30 \mathrm{~s}\right)$ in the increasing and decreasing temperature measurements, respectively. We can see that the variations of the data points are quite regular and actually they are consistent with the theoretical predictions. It is in- teresting to note that after RTA the room temperature grating period has been increased (by about $2.5 \mathrm{~nm}$ ), contrary to the case in Fig. 4. It implies that in this case the built thermal strains become compressive. Then, the gray triangles and $\mathrm{X}$ marks in Fig. 5 stand for the results after nitrogen-purged RTA $\left(800{ }^{\circ} \mathrm{C}\right.$ for $\left.30 \mathrm{~s}\right)$ and after $30 \mathrm{~s} 10 \% \mathrm{HF}$ dip in the increasing and decreasing temperature measurements, respectively. Now, the room temperature grating period is reduced back to the value before RTA. This implies that the existence of oxide causes tensile strain after RTA. Either tensile or compressive thermal strains mentioned above deserve further investigation.

In summary, we have fabricated silicon gratings with the period from 180 to $550 \mathrm{~nm}$ by using a laser ablation technique. In this technique, the interference fringe from a coherent and intense UV laser at $266 \mathrm{~nm}$ directly wrote periodical corrugations on a semiconductor surface. The temperature dependence of the grating period has been studied. It was found that RTA could release the thermal strains built during the melting and cooling process of laser ablation. Meanwhile, the removal of oxide may release strains in the other way. The measured temperature dependencies were consistent with the theoretical predictions.

This research was supposed by Grant Nos. NSC 862215-E-002-010, NSC 86-2215-E-002-011, and NSC 862212-M-002-024 from the National Science Council, The Republic of China.

${ }^{1}$ H. Hillmer, A. Grabmaier, H. L. Zhu, S. Hansmann, and H. Burkhard, J. Lightwave Technol. 13, 1905 (1995).

${ }^{2}$ C. H. Lin, Z. H. Zhu, Y. Qian, and Y. H. Lo, IEEE J. Quantum Electron. 32, 1752 (1996).

${ }^{3}$ Y. Shibata, S. Oku, Y. Kondo, and T. Tamamura, IEEE Photonics Technol. Lett. 8, 87 (1996).

${ }^{4}$ A. Sharon, D. Rosenblatt, and A. A. Friesem, Appl. Phys. Lett. 69, 4154 (1996).

${ }^{5}$ S. Yin, F. T. S. Yu, and S. Wu, IEEE Photonics Technol. Lett. 4, 894 (1992).

${ }^{6}$ B. J. Eggleton, P. A. Krug, and L. Poladian, Opt. Lett. 19, 877 (1994).

${ }^{7}$ O. Knacke, O. Kubaschewski, and K. Hesselmann, Thermochemical Properties of Inorganic Substances (Springer, New York, 1991).

${ }^{8}$ T. Suzuki, A. Mimura, and T. Ogawa, J. Electrochem. Soc. 124, 1776 (1977).

${ }^{9}$ Y. Okada and Y. Tokumaru, J. Appl. Phys. 56, 314 (1984). 